\title{
EXPLAINING COEXISTENCE OF NITROGEN FIXING AND NON-FIXING RHIZOBIA IN LEGUME-RHIZOBIA MUTUALISM USING MATHEMATICAL MODELING
}

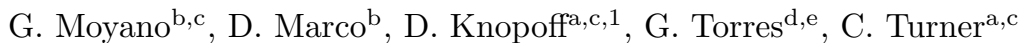 \\ ${ }^{a}$ CIEM-CONICET: Medina Allende s/n, Ciudad Universitaria, 5000, Córdoba, Argentina \\ ${ }^{b}$ FCEFyN-UNC, CONICET: Avda. Vélez Sársfield 1611, Ciudad Universitaria, 5000, \\ Córdoba, Argentina \\ ${ }^{c}$ FaMAF-UNC: Medina Allende s/n, Ciudad Universitaria, 5000, Córdoba, Argentina \\ ${ }^{d}$ FaCENA-UNNE: Avda. Libertad 5470, Campus Universitario, 3400, Corrientes, \\ Argentina \\ ${ }^{e}$ IMIT-CONICET: Avda. Libertad 5470, Campus Universitario, 3400, Corrientes, \\ Argentina
}

\begin{abstract}
In the mutualism established between legumes and soil bacteria known as rhizobia, bacteria from soil infect plants roots and reproduce inside root nodules where they fix atmospheric $N_{2}$ for plant nutrition, receiving carbohydrates in exchange. Host-plant sanctions against non $N_{2}$ fixing, cheating bacterial symbionts have been proposed to act in the legume-Rhizobium symbiosis, to preserve the mutualistic relationship. Sanctions include decreased rhizobial survival in nodules occupied by cheating rhizobia. Previously, a simple population model experimentally based showed that the coexistence of fixing and cheating rhizobia strains commonly found in field conditions is possible, and that the inclusion of sanctions leads to the extinction of cheating strains in soil. Here, we extend the previous model to include other factors that could complicate the sanction scenario, like horizontal transmission of symbiotic plasmids, turning non-nodulating strains into nodulating rhizobia, and competition between fixing and cheating strains for nodulation. In agreement with previous results, we show that plant populations persist even in the presence of cheating rhizo-
\end{abstract}

\footnotetext{
${ }^{1}$ damian.knopoff@unc.edu.ar
} 
bia without incorporating any sanction against the cheater populations in the model, under the realistic assumption that plants can at least get some amount of fixed $N_{2}$ from the effectively mutualistic rhizobia occupying some nodules. Inclusion of plant sanctions leads to the unrealistic extinction of cheater strains in soil. Our results agree with increasing experimental evidence and theoretical work showing that mutualisms can persist in presence of cheating partners.

Keywords: mutualism, cheating, legume-rhizobia symbiosis, host sanctions, mathematical modeling, agriculture.

\section{Introduction}

In the mutualism established between legumes and soil bacteria known as rhizobia, bacteria from soil infect plants roots and reproduce inside root nodules, where they fix atmospheric nitrogen for plant nutrition, receiving carbohydrates 5 in exchange. After nodule senescence, surviving rhizobia are released into the soil where, depending on their viability, they can maintain resident populations [1] and reinfect plants roots in the next growing cycle. This naturally occurring mutualism has been since long used in agriculture to add rhizobia to the crops as inoculants, to replace or at least reduce the use of nitrogen fertilizers, mainly as nitrates, which causes many environmental and human health problems. Field added nitrates leach to water contributing to eutrophication and the blooming of algae that diminishes oxygen water content, thus causing fish death. Nitrate contamination of drinking water is of great concern, since it can cause methemoglobinemia, especially in children [2]. Also, a nitrate metabolite, nitrosamine, is carcinogenic [3].

In the Rhizobium-legume mutualism, partner benefits are clear: plant receives nitrogen from Rhizobium bacteria inside the nodules and bacteria receive carbon compounds from the plant. However, strains of nodulating rhizobia that do not fix (or fix low) nitrogen are common in the soil and even coexisting in the same plant [4]. Nodulation by ineffective rhizobia is an example of "cheating" (receiving benefits but not reciprocating) by a partner mutualist [5]. This 
presents the problem of how can cooperation be maintained if partners pursuit only self-benefit? In the legume-rhizobia symbiosis, decreased nodular rhizobial viability and/or early nodule senescence have been proposed as plant host sanctions against non fixing, cheating rhizobia $[6,7,8]$. However, in a previous work combining experiments and mathematical modeling no evidence of plant host sanctions was found [9]. In that first approach, the ecological stability of Rhizobium-legume symbiosis, when "cheating" strains were present, was analyzed using a population dynamics model with and without the inclusion of plant host sanctions. Here, we extend the previous model [9] to include other factors that confer more realistic conditions, and could complicate the sanction scenario, like horizontal transmission of symbiotic ( sym) plasmids, turning nonnodulating strains into nodulating rhizobia [10], and competition between fixing and cheating strains for nodulation [11]. Transfer of plasmids conferring nodulation abilities would lead to changes in the frequency of fixing and non-fixing rhizobia population both in the soil and coming from nodules. Competition in our modeling context would lead to changes in the rhizobial population densities, depending on which strain, fixing or non-fixing, is given the competitive advantage.

The inclusion of these two factors will allow for a new and deeper understanding of the Rhizobium-legume symbiotic persistence in the rhizobial cheating and host-sanction context.

\section{Model development and biological background}

The model is based on an experimental approach allowing to directly and 45 unambiguously testing a potential sanction from the plant to a true cheating rhizobium sharing the same plant with an effective strain [12]. Details of the biological framework of the model are given in [9]. Briefly, the model formulation is based on several biological features of the mutualistic system and the following assumptions, either checked or supported by experimental tests:

- Sym plasmid is the only factor that confers nodulation ability and fix- 
ing capacity (fix + or fix-). It can be transferred from plasmid-carrying bacteria (fix + and fix-) to recipient bacteria without plasmid (plasmidfree bacteria) in the soil [10]. A single bacterium can carry only one sym plasmid (fix + or fix-) at a time.

- Fixing and non-fixing bacterial strains differ in their $N_{2}$ fixing ability and competition levels for nodule initiation [11, 12]

- Nodules are initiated and occupied by a single bacterium of either fixing or non-fixing strain [13].

- Nodules are occupied to their carrying capacity, and are functionally equivalent and metabolically independent of each other [6].

- Fixing and non-fixing nodules can develop and coexist in the same plant [12].

- There is a minimum number of fixing nodules per plant needed to develop and produce seeds [12].

- At the end of each annual cycle nodules undergo senescence and release surviving bacteria into the soil [1].

- The number of bacteria coming to the soil from nodules occupied by fixing and non-fixing bacteria can vary if plant sanctions are assumed [6].

- The time scale is one year, assuming an annual plant and a slow rhizobial turnover in soil [1].

We modeled the mutualistic plant-rhizobia system described above using logistic mappings. Equations represent the plant population, the populations of free bacteria living in the soil closely surrounding the root, fixing and nonfixing bacteria, and plasmid-free bacteria, and bacteria inside nodules. Fig. 1 shows a scheme of the model. Details of the biological framework of the model are given in [9]. Briefly, we describe the fixing and non-fixing bacteria and recipient bacteria without plasmid populations in soil by two coupled logistic 


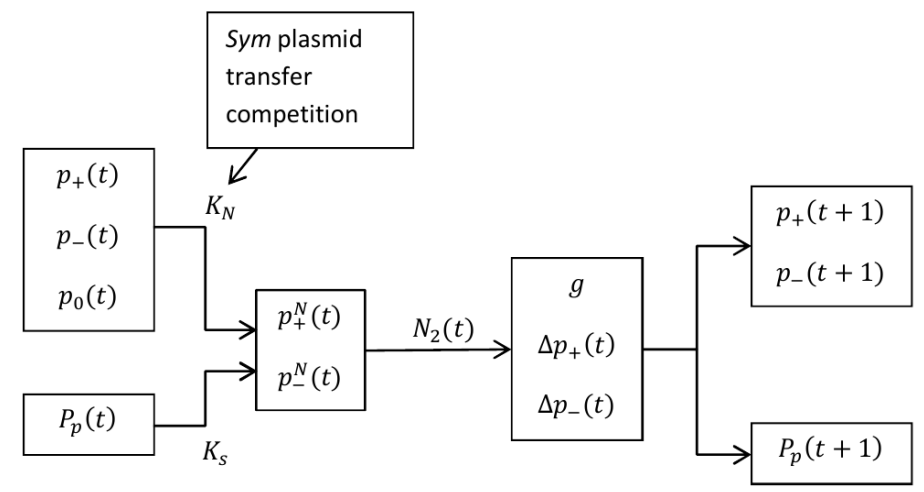

Figure 1: Schematic structure of the model dynamics in a single iteration. Initial values of plant and bacteria populations $\left(P_{p}\right.$ for plants; $p_{+}, p_{-}$and $p_{0}$ for bacteria in soil) set the number of nodules formed after sym plasmid transfer and competition between fix + and fixrhizobia, and so the values of bacteria in nodules $\left(p_{+}^{N}\right.$ and $\left.p_{-}^{N}\right)$ to be released in soil after nodule senescence. The bacteria in nodules provide $N_{2}$ to the plants and the new populations are calculated based on the produced seeds $(g)$ and the released bacteria $\left(\Delta p_{+}\right.$and $\left.\Delta p_{-}\right)$. The total number of nodules generated by bacterial strains $K_{N}$ and $K_{s}$ denotes the total root colonisable sites for nodule initiation.

maps, modified to take into account the bacteria coming into the soil from the senescent nodules (fix + , fix-), and already in the soil (plasmid-free bacteria):

$$
\begin{gathered}
p_{i}(t+1)=\left(p_{i}(t)+\Delta p_{i}(t)\right)\left(1+r_{i}^{s}\left(1-\frac{P_{T}(t)}{\delta_{s}}\right)\right)+\beta p_{0}(t) p_{i}(t)-\tau p_{i}(t), \quad i=+,- \\
p_{0}(t+1)=p_{0}(t)\left(1+r_{0}^{s}\left(1-\frac{P_{T}(t)}{\delta_{s}}\right)\right)-\left(p_{0}(t) \beta-\tau\right)\left(p_{+}(t)+p_{-}(t)\right), \\
P_{T}(t)=p_{+}(t)+\Delta p_{+}(t)+p_{-}(t)+\Delta p_{-}(t)+p_{0}(t)
\end{gathered}
$$

where $p_{i}$ describes the bacteria population densities in soil, $i=+,-, 0$ indicates fixing and non-fixing bacteria, and plasmid-free bacteria respectively, and $P_{T}$ is the total bacteria population density (i.e., fixing plus non-fixing plus plasmidfree bacteria) in soil. The parameter $\delta_{s}$ stands for the carrying capacity of the soil close to roots in absence of nodulation. An effective carrying capacity greater than $\delta_{s}$ is set when plants release bacteria at the end of nodulation process. Parameter $\beta$ stands for the probability of horizontal sym plasmid 
transfer from fix + or fix- bacteria to plasmid-free bacteria and its value was

90 set at $10^{-6}$ events per recipient cell [14]. Constant $\tau$ is the probability of sym plasmid loss and its value was set at $10^{-3}$ events per sym plasmid carrying cell $[15]$.

The parameter $r_{i}^{s}$ represents the intrinsic reproduction rate of each population in the soil close to roots, assumed to be equal $r_{+}^{s}=r_{-}^{s}=r_{0}^{s}=r^{s}$.

95 The total number of the surviving bacteria that returns to the soil at time $t$ is calculated as $\Delta p_{i}(t)$. Dynamics of $p_{0}$ occurs exclusively in the soil, and is affected by sym plasmid transfer $(\beta)$ and plasmid loss $(\tau)$. Plants are not able to differentiate fixing from non-fixing bacteria during the root colonization process [11]. If we assume no selection of rhizobia by plants inside nodules, the number $f_{i}$ of surviving bacteria of each type released from a nodule will be the same for fixing and non fixing bacteria (about $10^{-4}$ of the carrying capacity of a nodule). If we consider that plants can recognize and sanction the non-fixing rhizobia, the surviving number of non-fixing rhizobia would be lower than the surviving number of the fixing ones $[6,7,16]$. In this last case, we allowed the number $f_{i}$ of surviving bacteria of each type to be different, i.e.

$$
\Delta p_{i}(t)=\frac{f_{i} \delta_{n}}{m_{s}} K_{i}^{N}(t), \quad i=+,-,
$$

where $\delta_{n}$ is the carrying capacity of each nodule type, $m_{s}$ is the mass of soil per hectare associated to the crop and $f_{+}=f, f_{-}=f(1-\sigma)$. The parameter $\sigma$ represents the sanction intensity that plant applies to the non-fixing bacteria. Its value goes from 0 to 1 , where $\sigma=0$ represents the case without sanction.

The number of nodules generated by each type of bacterial strain is $K_{i}^{N}(t)$, and it represents a fraction of the total root colonisable sites for nodule initiation $\varphi K_{s}(t)$. We allow for both rhizobial strains to vary in their ability to colonize the root and initiate nodules.

If $p_{+}(t)+p_{-}(t) \geq p_{m}$ then $K_{+}^{N}$ and $K_{-}^{N}$ are defined as the solution of the system:

$$
\frac{K_{+}^{N}}{K_{-}^{N}}=C\left(\frac{p_{+}}{p_{-}}\right)^{w}
$$




$$
K_{+}^{N}+K_{-}^{N}=\varphi K_{s}
$$

where $\varphi$ is the fraction of nodulation sites effectivelly forming nodules. On the other hand, if $p_{+}(t)+p_{-}(t)<p_{m}$ then $K_{+}^{N}=K_{-}^{N}=0$. Here $K_{s}(t)=n P_{p}(t)$ is the number of sites available for nodulation, where $n$ is the average number of nodules per plant considered proportional to the plant population $P_{p}(t)$ (number of plants per hectare), the threshold $p_{m}$ is the minimum bacteria population per gram of soil needed to trigger the nodulation process, $C$ and $w$ are the competition coefficient and exponent respectively in Amarger's equation [17]. When $C=1$ and $w=1$ there is no competition between fix + and fix - strains for nodule initiation, and so the nodulation is assumed to be at random. If $C>1$ then strain fix + is more competitive. For simulations, $C$ values were taken between 0.8 and 1.8 [18], and $w$ was fixed at 0.4 [17]. The maps representing the free bacteria in the soil are coupled to the plant system through the factor $K_{s}$ (total root colonisable sites for nodule initiation).

The plant population is defined by

$$
P_{p}(t+1)=\delta_{p}\left[1-\exp \left(-g(t) \frac{\left|\log \left(1-\Pi_{g}\right)\right| P_{p}(t)}{\delta_{p}}\right)\right] .
$$

Briefly, $\delta_{p}$ is the plant carrying capacity of the field, $\Pi_{g}$ is the probability that a seed germinates and develops into an adult plant, and $g$ is the mean number of seeds produced by a plant in an annual crop, that is assumed to depend on the number of nodules colonized by fixing bacteria and the plant population itself, i.e. $g(t)=h\left(K_{+}^{N}(t), P_{p}(t)\right)$ for a given function of two variables $h$.

The number of seeds depends on the amount of available nitrogen for plants at time $t$. The more nitrogen is available to the plants, the more seeds they produce. We will assume that the amount of nitrogen a plant can obtain depends only on the number of nodules colonized by fixing bacteria; hence, $h$ will be a monotonously increasing function of $K_{+}^{N}$. It is also reasonable to assume that there is a maximum number of seeds a plant can produce, denoted as $G$. On the other hand, if there is not enough nitrogen to support the plant seed production, the number of seeds should drop to zero. This means that there is a minimum 
number of nodules $K_{0}$ colonized by fixing bacteria required to produce seeds. Notice that plants that not reach $K_{0}$ do not complete their development and ultimately die from nitrogen starvation [12].

All the previous assumptions can be modeled by the following expression

$$
h\left(K_{+}^{N}, P_{p}\right)=\left\{\begin{array}{rc}
G \tanh \left(\frac{K_{+}^{N}-K_{0} P_{p}}{G P_{p}}\right), & \text { if } K_{+}^{N}-K_{0} P_{p}>0 \\
0, & \text { otherwise. }
\end{array}\right.
$$

If the sum of bacterial populations $p_{+}+p_{-}$is below the value of $p_{m}$ then it does not interact with the plant system and the plant dynamic is entirely given by its own logistic dynamic in the soil.

\section{Model analysis and results}

We compare the behavior of the model looking at bacteria and plant population dynamics in soil, for different values of $C$ (the competition coefficient) and $\sigma=0$ (without sanction), $\sigma=0.5$ (moderate sanction) and $\sigma=1$ (total sanction). Sensitivity analysis showed that changing the value of $w$ (slope of the competition equation) does not significantly influence population dynamics of strains and plants. In Table 1 we show the values of the parameters that were used through the numerical simulations. Initial conditions were: $p_{0}=200$, $p_{+}=400, p_{-}=400$ and $P_{p}=20000$.

Assuming no sanction $(\sigma=0)$, plants are unable to discriminate among fix + and fix - rhizobia, and so there is no strain selection. Hence, in our model $f_{+}=f_{-}$, i.e. the number of surviving bacteria that returns to the soil is the same for both strains. In all simulations we consider the case in which $p_{+}(0)+p_{-}(0) \geq p_{m}$, i.e. there is enough rhizobia in soil to elicit nodulation. In all the considered cases without sanction, the population of free-plasmid bacteria $p_{0}$ remains in the system even with very low values, reaching an equilibrium in which the plasmid transfer rate and the plasmid loss rate are balanced. 


\begin{tabular}{|c|c|c|}
\hline Parameter & Value & Description \\
\hline$r_{i}^{s}$ & $10^{-1}$ & $\begin{array}{l}\text { Intrinsic rate of growth of bacteria in the } \\
\text { soil }(i=+,-, 0)[1] .\end{array}$ \\
\hline$\delta_{s}$ & $10^{6} g^{-1}$ & $\begin{array}{l}\text { Soil bacterial carrying capacity (per } g \text { of } \\
\text { soil) }[1] \text {. }\end{array}$ \\
\hline$\beta$ & $10^{-6}$ & $\begin{array}{l}\text { Probability of sym plasmid transfer (event } \\
\text { per recipient cell). }[14]\end{array}$ \\
\hline$\tau$ & $10^{-3}$ & $\begin{array}{l}\text { Probability of sym plasmid loss (event per } \\
\text { sym plasmid carrying cell) [15]. }\end{array}$ \\
\hline$C$ & 0.8 to 1.8 & Competition coefficient [18]. \\
\hline$w$ & 0.4 & Competition coefficient [17]. \\
\hline$\varphi$ & $0-1$ & $\begin{array}{l}\text { Fraction of nodulation sites effectively } \\
\text { forming nodules [12]. }\end{array}$ \\
\hline$\delta_{n}$ & $10^{6}$ & $\begin{array}{l}\text { Nodule's carrying capacity (bacteria per } \\
\text { nodule) }[12] .\end{array}$ \\
\hline$f$ & $10^{-4}$ & $\begin{array}{l}\text { Surviving bacteria released from nodule } \\
{[12] \text {. }}\end{array}$ \\
\hline$\delta_{p}$ & $2 \times 10^{5} \mathrm{Ha}^{-1}$ & Plants' field carrying capacity [9]. \\
\hline$m_{s}$ & $1.5 \times 10^{5} \mathrm{gHa}^{-1}$ & $\begin{array}{l}\text { Soil mass per hectare associated to the } \\
\text { plant population [9]. }\end{array}$ \\
\hline$n$ & 45 & Typical number of nodules per plant [12]. \\
\hline$K_{0}$ & $0.15 \times n$ & $\begin{array}{l}\text { Minimum number of fixing nodules per } \\
\text { plant needed for seed production [12]. }\end{array}$ \\
\hline$G$ & 55 & $\begin{array}{l}\text { Maximum number of viable seeds pro- } \\
\text { duced per plant }[9] \text {. }\end{array}$ \\
\hline$\Pi_{g}$ & 0.69 & $\begin{array}{l}\text { Probability of a viable seed reaching the } \\
\text { adult stage [9]. }\end{array}$ \\
\hline$\sigma$ & $0-1$ & $\begin{array}{l}\text { Sanction intensity } 0=\text { No sanction, } 1= \\
\text { maximum sanction. }\end{array}$ \\
\hline$p_{m}$ & $0-10^{2} g^{-1}$ & $\begin{array}{l}\text { Minimum bacteria population per } g \text { of soil } \\
\text { needed to trigger the nodulation process } \\
{[12] \text {. }}\end{array}$ \\
\hline
\end{tabular}

Table 1: Model parameters 
The populations of plasmid bearing strains $p_{+}$and $p_{-}$show a fast growth during the first initial cycles, to reach equilibrium at about 500 years, with relative values of $p_{+}$and $p_{-}$depending on the competition coefficient $C$. Notice that increasing values of $C$ show increasing relative population of $p_{+}$with respect to $p_{-}$, although both populations coexist in time anyways. Indeed, we can see that rising the competitive ability of the fix + strain $(C=0.8$ to 1.8$)$, has the effect of increasing its population even more in at least one order of magnitude (see Fig. 2a, 2b and 2c). Plant population stabilizes at $2 \times 10^{5}$ plants/hectare within a few cycles, and it is not influenced by changes in strains' competitive abilities.

Notice that the fact that plant population converges to the value $\delta_{p}=2 \times 10^{5}$ can be proved analytically, by showing that $\delta_{p}$ is a fixed point of Eq. 7 ; in other words, that if the carrying capacity $\delta_{p}$ is reached at time $t$ then the population of plants stabilizes at this value for later times. Indeed, let us suppose that there exists a time $t_{*}$ such that $P_{p}\left(t_{*}\right)=\delta_{p}$ (i.e. the carrying capacity is reached at $t_{*}$ ). Then, since right hand side of equation 7 is increasing we have $P_{p}\left(t_{*}+1\right) \geq P_{p}\left(t_{*}\right)=\delta_{p}$. But we also have that $P_{p}(t) \leq \delta_{p}$ for all $t$. Consequently, $\delta_{p} \leq P_{p}\left(t_{*}+1\right) \leq \delta_{p}$, which let us conclude that $P_{p}\left(t_{*}+1\right)=\delta_{p}$. Analysis of steady states of bacterial populations is quite more challenging and it is discussed in the Supplementary material.

Allowing for moderate sanction $(\sigma=0.5)$, i.e. half of the nodules prevented from releasing bacteria into the soil, the overall behavior of the bacteria populations is qualitatively similar to the case without sanction. The advantage of $p_{+}$over $p_{-}$is increased even with the lower $C$ value. A stable coexistence between the strains is reached at times similar to those without sanction (see Fig. 2d, 2e and 2f). Plant population stabilizes at $2 \times 10^{5}$ plants/hectare within a few cycles, and again, it is not influenced by changes in strains' competitive abilities.

Considering total sanction $(\sigma=1)$, plants do not allow the non-fixing bacteria inside the nodules to come into the soil. While the fix - rhizobia go extinct (less than one bacterium per gram of soil) very early, the fixing bacterial popu- 

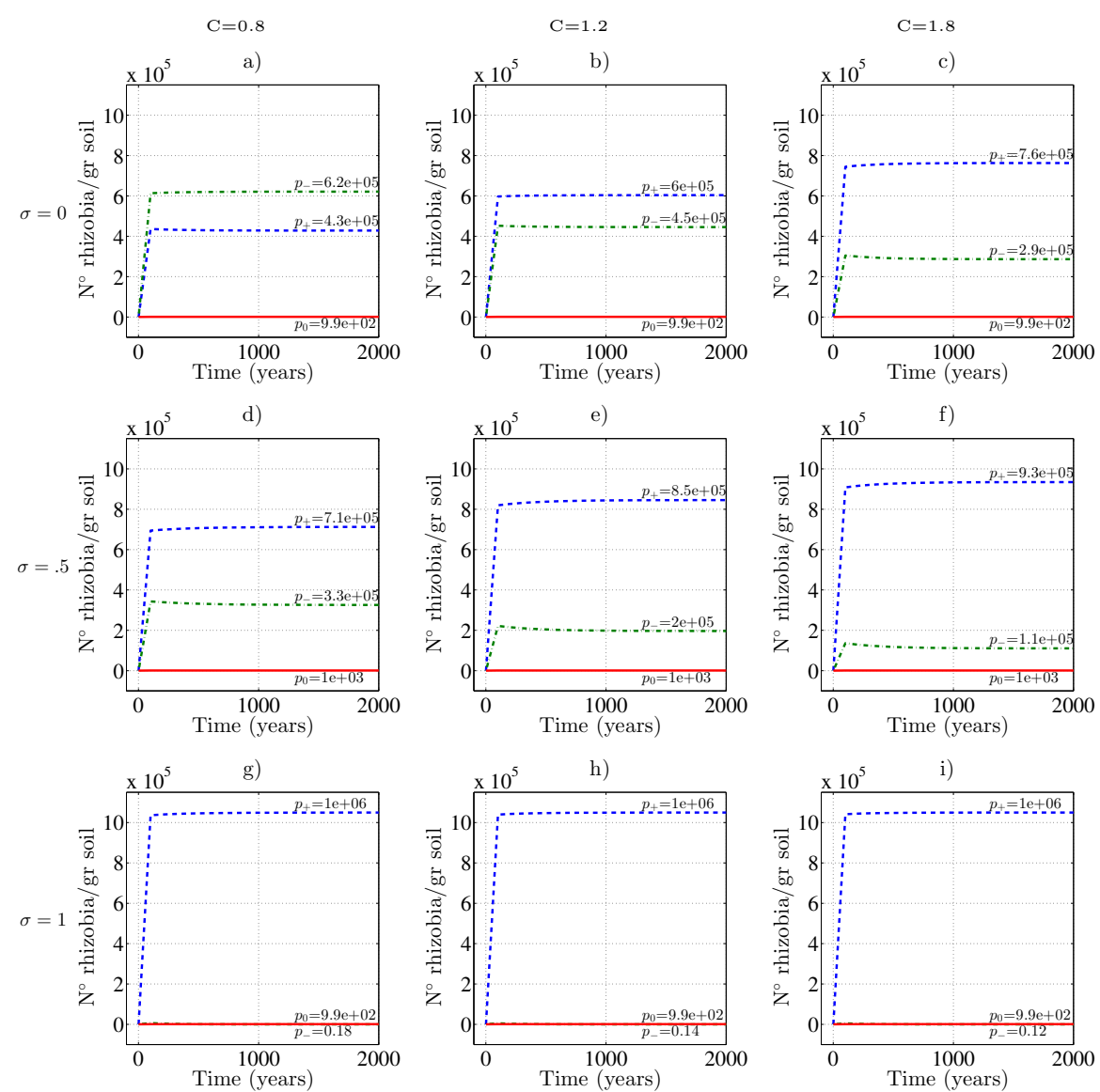

Figure 2: Simulation results showing dynamics of strains populations in soil, fix $+\left(p_{+}\right)$, fix$\left(p_{-}\right)$and plasmid-free rhizobia $\left(p_{0}\right)$ under different sanction $(\sigma)$ and competitive ability $(C)$ scenarios. First row corresponds to no sanction $(\sigma=0)$, second row corresponds to moderate sanction $(\sigma=0.5)$ and third row corresponds to total sanction $(\sigma=1)$. First column corresponds to $C=0.8$, second column corresponds to $C=1.2$ and third column corresponds to $C=1.8$. 
lations grow to higher levels due to the reinsertion of the bacteria coming from

the senescent nodules. Varying competition ability for the fixing strains does not exert any effect in a scenario of total sanction (see Fig. 2g, 2h and 2i). This means that, in the long term when the plant population apply extreme sanctions, only fixing rhizobia will be present in the system. As in the previous cases, plant population stabilizes at $2 \times 10^{5}$ plants/hectare within a few cycles, and it is not influenced by changes in strains' competitive abilities.

Fig. 3 shows the ratio of fix + and fix - populations varying the strains' competitive abilities under different sanction scenarios. From here we can observe that the large time behaviors of the two populations are very similar with no sanction or moderate sanction while the influence of a greater competitive ability of the fix + strain is reflected in a slight ratio increase (notice that color stripes become wider as $\mathrm{C}$ increases). However, the application of total sanction renders a higher ratio increase favouring the fix + strain and showing the extinction of the fix- strain.

Sensitivity analysis performed to analyze the influence of horizontal transfer of the sym plasmid, performing simulations without sym transfer under the same conditions of sanction and competition, showed that the overall system behaviour is maintained. However, there is a small quantitative effect, with the population of $p_{0}$ increasing (since there is no flux from $p_{0}$ to $p_{+}$and $p_{-}$), and the populations of $p_{+}$and $p_{-}$decreasing (because there is no influx from $p_{0}$ ). The effect is small since the rate of sym plasmid transfer is low, according to the used experimental values (See Fig. S1 Supplementary material).

\section{Discussion and conclusions}

Our results are in agreement with previous results of the model [9], showing that a simple population model including strain competition can explain the coexistence of fixing and non-fixing, cheating rhizobia strains, as it commonly occurs in field conditions. Also as previously found, inclusion of plant sanctions is not required to explain strain coexistence. Plant populations are able to 


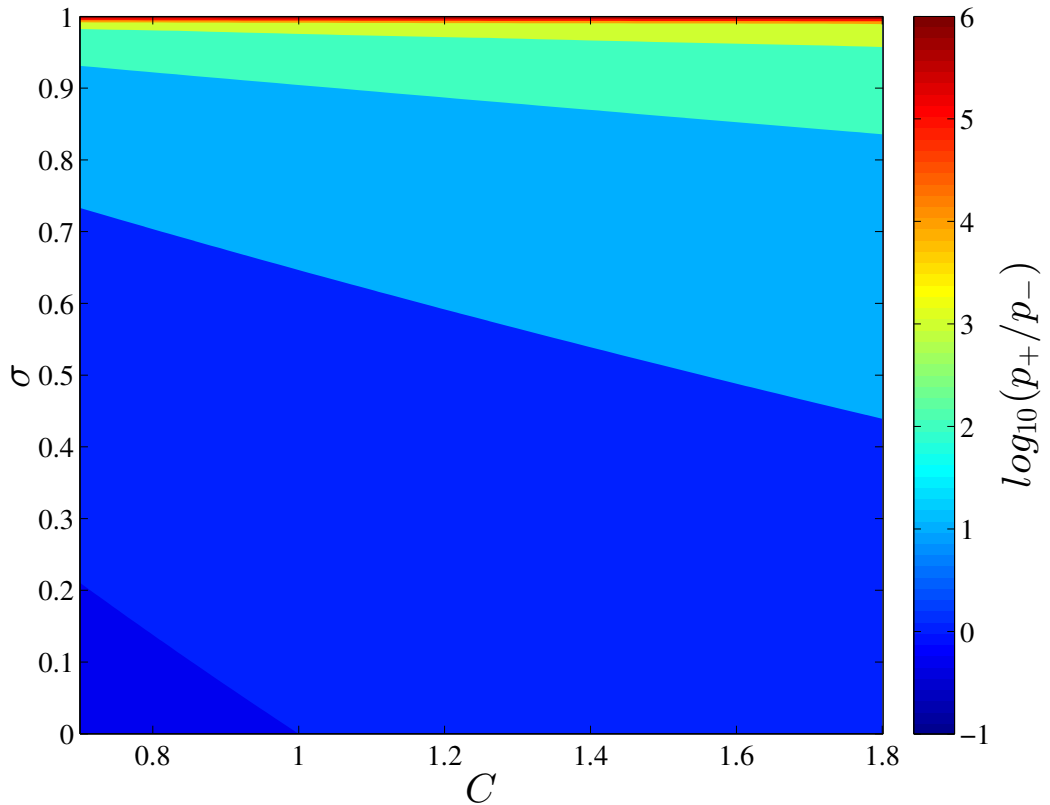

Figure 3: Simulation results showing the ratio of strains populations in soil, fix $+(p+)$, fix$(p-)$ under different sanction scenarios and competition coefficients, for large time. Horizontal axis represents competition coefficient $(C)$ values from 0.8 to 1.8 , vertical axis corresponds to sanction values $(\sigma)$ in the interval from 0 to 1 while the intensity of the colors stands for the ratio of strain populations at final time, represented as the $\log _{10}\left(p_{+} / p_{-}\right)$. 
maintain a stable equilibrium provided they get fixing rhizobia needed to provide a minimum $N_{2}$ amount, despite being cheated by non-fixing rhizobia.

The assumption of no different competitive abilities between strains for nodulation made in the previous version of the model was relaxed and instead, fixing and non-fixing were allowed to compete for nodulation sites. We explored a range of competition coefficient values, changing competitive advantage from fix - to fix + strains. This is an interesting scenario for agricultural practices, since a common and problematic situation in crops occurs, when in spite of field inoculation with highly efficient rhizobia strains, after a few years nodulation becomes produced by more competitive but less efficient or even non-fixing strains residing in soil $[11,4,19]$. Our results showed that even slight improvements in nodulation competitiveness of the fixing strains used in inoculants could result in significant raises in fixing strains populations in soil with the resulting reduction in the use of nitrogen fertilizers. The inclusion of horizontal transfer of the symbiotic plasmid, conferring nodulation ability to soil rhizobia, is quite challenging since finding experimental values is difficult. We based our parameter value for $\beta$ on the experimental results reported by Kinkle and Schmidt [14], who recorded horizontal transfer of a symbiotic plasmid between Sinhorizobium fredii and Rhizobium leguminosarum strains nodulating pea, under different conditions (sterile and non sterile soil, humidity, temperature and inoculum amount).

We found that horizontal transfer of the sym plasmid exerts a quantitative effect on rhizobia in the soil, although this effect is small since the rate of sym plasmid transfer is low, according to the used experimental values [14]. Transfer of sym plasmid does not so far represent an important factor in the context of the mutualistic system dynamics explored in this work, where pre-existence in the soil of nodulating rhizobia is assumed (like in agricultural systems artificially inoculated). However, since transmission of sym plasmid confers the ability of nodulating and eventually fixing nitrogen, it may represent an important factor in fields harbouring non-nodulating rhizobia that could eventually became nodulating (and fixing) through sym plasmid transfer from inoculated 
or invading nodulating strains [10].

In this modelling context, plants receive nitrogen only from rhizobia fixation, in consequence, plants that not reach a minimum amount of nitrogen from fixing nodules do not complete their development and ultimately die from nitrogen starvation (supported by experimental results, [12]). So, the inclusion in the model of sanctions from plants to non-fixing rhizobia [6], as well as in the previous version of the model, was made in the context of plants reaching a minimum number of fixing nodules. What it can be concluded from this scenario is that, provided the plants reach a minimum number of fixing nodules and applying moderate sanctions, the cheating and the fixing strains coexist in the system and the plant population survives and stabilises over time. Coexistence is also obtained with no sanctions, so we conclude that considering the host sanction hypothesis is not necessary to explain coexistence of cheating and fixing strains. Furthermore, stronger sanctions as proposed by Denison [6], lead to the disappearance of cheating strains from the system. However, field evidence shows that cheating strains persist in soil and chronically hamper crop yields [11].

From a theoretical point of view, our results support the idea that cheating does not necessarily endanger rhizobia-legume mutualism and that a gradient from mutualism to parasitism can be found in nature [5]. There is an increasing wealth of theoretical and empirical evidence that cheating is widespread and punishment is rarely applied to defective mutualistic partners, and that other factors like herbivory can influence mutualisms [20, 21]. Furthermore, costs of cheating in rhizobia-legume mutualism may not be as high as assumed if the host is still able of obtaining benefits from other mutualistic partners, as in co-infected plants that are common in field $[4,19]$. This situation will be incorporated in the model in further versions, allowing for co-occupation of the same nodule by strains with different fixation abilities. About $20 \%$ of total nodules are usually co-occupied at field by different rhizobial strains in artificial inoculations [22]. We expect the effects of co-occupation of nodules by nonfixing rhizobia will be diluted by fixing rhizobia occupying the same nodule, 
thus not favoring plant sanctions.

\section{Acknowledgments}

This work was partially supported by grants from CONICET (2015-2017), CONICET (PIP 11420090100102, PIP 11220110100050), SECYT-UNC (20152016) and ANPCyT (PICT-2015-1066).

Authors would like to thank the Editor and the anonymous reviewers for the careful reading of the manuscript and their valuable comments.

\section{References}

[1] P. R. Hirsch, Population dynamics of indigenous and genetically modified rhizobia in the field, New Phytologist 133 (1) (1996) 159-171.

[2] F. R. Greer, M. Shannon, et al., Infant methemoglobinemia: the role of dietary nitrate in food and water, Pediatrics 116 (3) (2005) 784-786.

[3] V. M. Craddock, A. R. Henderson, Effect of n-nitrosamines carcinogenic for oesophagus on o6-alkyl-guanine-dna-methyl transferase in rat oesophagus and liver, Journal of Cancer Research and Clinical Oncology 111 (3) (1986) $229-236$

[4] P. Singleton, J. Tavares, Inoculation response of legumes in relation to the number and effectiveness of indigenous Rhizobium populations, Applied and Environmental Microbiology 51 (5) (1986) 1013-1018.

[5] J. L. Bronstein, The exploitation of mutualisms, Ecology Letters 4 (3) (2001) 277-287.

[6] R. F. Denison, Legume sanctions and the evolution of symbiotic cooperation by rhizobia, The American Naturalist 156 (6) (2000) 567-576.

[7] E. T. Kiers, R. A. Rousseau, S. A. West, R. F. Denison, Host sanctions and the legume-rhizobium mutualism, Nature 425 (6953) (2003) 78-81. 
[8] E. T. Kiers, R. A. Rousseau, R. F. Denison, Measured sanctions: legume hosts detect quantitative variation in rhizobium cooperation and punish accordingly, Evolutionary Ecology Research 8 (6) (2006) 1077-1086.

[9] D. E. Marco, J. P. Carbajal, S. Cannas, R. Pérez-Arnedo, Á. Hidalgo-Perea, J. Olivares, J. E. Ruiz-Sainz, J. Sanjuán, An experimental and modelling exploration of the host-sanction hypothesis in legume-rhizobia mutualism, Journal of Theoretical Biology 259 (3) (2009) 423-433.

[10] J. T. Sullivan, H. N. Patrick, W. L. Lowther, D. B. Scott, C. W. Ronson, Nodulating strains of Rhizobium loti arise through chromosomal symbiotic gene transfer in the environment, Proceedings of the National Academy of Sciences 92 (19) (1995) 8985-8989.

[11] N. Amarger, Competition for nodule formation between effective and ineffective strains of Rhizobium meliloti, Soil Biology and Biochemistry 13 (6) (1981) 475-480.

[12] D. E. Marco, R. Pérez-Arnedo, Á. Hidalgo-Perea, J. Olivares, J. E. RuizSainz, J. Sanjuán, A mechanistic molecular test of the plant-sanction hypothesis in legume-rhizobia mutualism, Acta Oecologica 35 (5) (2009) 664667.

[13] D. J. Gage, Infection and invasion of roots by symbiotic, nitrogen-fixing rhizobia during nodulation of temperate legumes, Microbiology and Molecular Biology Reviews 68 (2) (2004) 280-300.

[14] B. K. Kinkle, E. L. Schmidt, Transfer of the pea symbiotic plasmid pJB5JI in nonsterile soil, Applied and Environmental Microbiology 57 (11) (1991) $3264-3269$.

[15] B. T. Lau, P. Malkus, J. Paulsson, New quantitative methods for measuring plasmid loss rates reveal unexpected stability, Plasmid 70 (3) (2013) 353361. 
[16] S. A. West, E. T. Kiers, E. L. Simms, R. F. Denison, Sanctions and mutualism stability: why do rhizobia fix nitrogen?, Proceedings of the Royal Society of London B: Biological Sciences 269 (1492) (2002) 685-694.

[17] N. Amarger, J. P. Lobreau, Quantitative study of nodulation competitiveness in Rhizobium strains, Applied and Environmental Microbiology 44 (3) (1982) 583-588.

[18] S. Okazaki, K.-I. Yuhashi, K. Minamisawa, Quantitative and timecourse evaluation of nodulation competitiveness of rhizobitoxine-producing

$350 \quad$ Bradyrhizobium elkanii, FEMS Microbiology Ecology 45 (2) (2003) 155160.

[19] D. Dowling, W. Broughton, Competition for nodulation of legumes, Annual Reviews in Microbiology 40 (1) (1986) 131-157.

[20] J. Leigh, et al., The evolution of mutualism, Journal of Evolutionary Biology 23 (12) (2010) 2507-2528.

[21] A. K. Simonsen, J. R. Stinchcombe, Herbivory eliminates fitness costs of mutualism exploiters, New Phytologist 202 (2) (2014) 651-661.

[22] B. G. Rolfe, P. M. Gresshoff, Rhizobium trifolii mutant interactions during the establishment of nodulation in white clover, Australian Journal of

$360 \quad$ Biological Sciences 33 (4) (1980) 491-504. 


\section{Supplementary material}

\section{Analysis of the influence of horizontal transfer}

This supplementary section is devoted to the sensitivity analysis performed to analyze the influence of horizontal transfer of the sym plasmid, performing simulations without sym transfer under the same conditions of sanction and competition. Consequently, in the following we set $\beta=0$ while the rest of parameter values are the same as those employed in the simulations showed in Fig. 2. It is worth comparing Fig. S1 with Fig. 2, to see that the overall system behaviour is maintained. However, there is a small quantitative effect, with the population of $p_{0}$ increasing (since there is no flux from $p_{0}$ to $p_{+}$and $p_{-}$), and the populations of $p_{+}$and $p_{-}$decreasing (because there is no influx from $p_{0}$ ). The effect is small since the rate of sym plasmid transfer is low, according to the used experimental values.

\section{Analysis of steady states of bacterial populations}

Following the reasonings for calculating the stationary steady state for plant population $P_{p}(t)$ in Section 3, let us now suppose that there exists a sufficiently large time $t_{*}$ such that bacterial populations reach their respective steady states, namely

$$
p_{+}\left(t_{*}+1\right)=p_{+}\left(t_{*}\right), \quad p_{-}\left(t_{*}+1\right)=p_{-}\left(t_{*}\right), \quad p_{0}\left(t_{*}+1\right)=p_{0}\left(t_{*}\right) .
$$

Let us define variables $x=p_{+}\left(t_{*}\right), y=p_{-}\left(t_{*}\right)$ and $z=p_{0}\left(t_{*}\right)$ as the steady unknown states to be determined. Notice that for $t \geq t_{*}$ the steady state has been reached and thus system (5)-(6) gives

$$
K_{-}^{N}(t)=\frac{\varphi K_{s}}{C\left(\frac{x}{y}\right)^{w}+1}, \quad K_{+}^{N}(t)=\varphi K_{s}\left(1-\frac{1}{C\left(\frac{x}{y}\right)^{w}+1}\right),
$$

whith $K_{s}=n \delta_{p}$ (notice that $\delta_{p}=P_{p}\left(t_{*}\right)$ ). Replacing into equation (4) for $\Delta p_{+}$ and $\Delta p_{-}$we get

$$
\Delta p_{+}(t)=\frac{f_{+} \delta_{n} \varphi K_{s}}{m_{s}}\left(1-\frac{1}{C\left(\frac{x}{y}\right)^{w}+1}\right),
$$



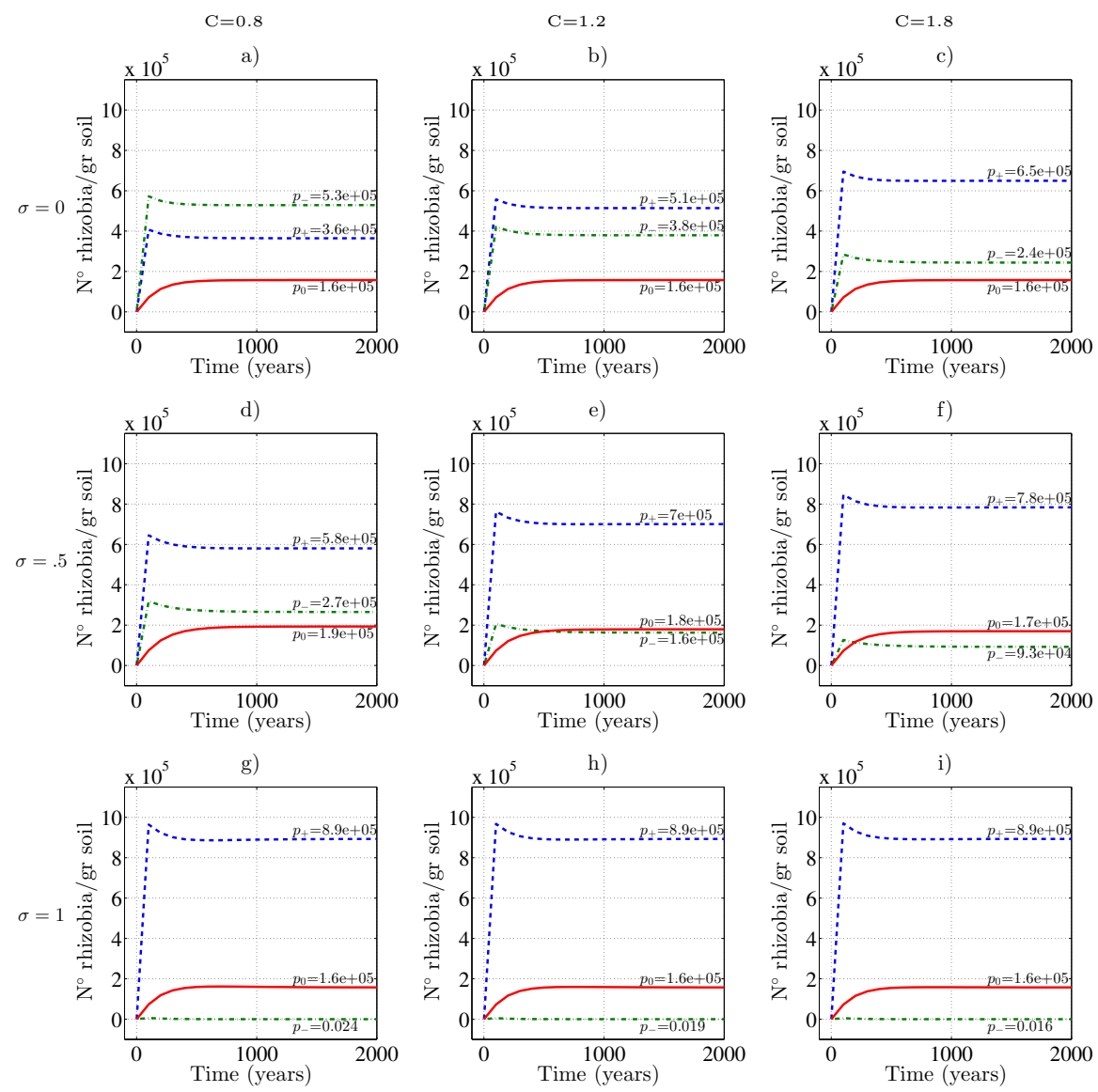

Figure S1: Simulation results without sym transfer, showing dynamics of strains populations in soil, fix $+\left(p_{+}\right)$, fix- $\left(p_{-}\right)$and plasmid-free rhizobia $\left(p_{0}\right)$ under different sanction $(\sigma)$ and competitive ability $(C)$ scenarios. First row corresponds to no sanction $(\sigma=0)$, second row corresponds to moderate sanction $(\sigma=0.5)$ and third row corresponds to total sanction $(\sigma=1)$. First column corresponds to $C=0.8$, second column corresponds to $C=1.2$ and third column corresponds to $C=1.8$.

$$
\Delta p_{-}(t)=\frac{f_{-} \delta_{n} \varphi K_{s}}{m_{s}}\left(\frac{1}{C\left(\frac{x}{y}\right)^{w}+1}\right) .
$$


Now, using Eqs. (1)-(3) and conditions in Eq. (9) we obtain

$$
\begin{aligned}
(x+A G(x, y))\left[1+r\left(1-\frac{1}{\delta_{s}} H(x, y, z)\right)\right]+x(\beta z-\tau-1) & =0, \\
(y+B F(x, y))\left[1+r\left(1-\frac{1}{\delta_{s}} H(x, y, z)\right)\right]+y(\beta z-\tau-1) & =0, \\
z\left[1+r\left(1-\frac{1}{\delta_{s}} H(x, y, z)\right)\right]+(\tau z-\beta)(x+y)-z & =0,
\end{aligned}
$$

where $A=\frac{f_{+} \delta_{n} \varphi K_{s}}{m_{s}}, B=\frac{f_{-} \delta_{n} \varphi K_{s}}{m_{s}}, F(x, y)=\frac{1}{C\left(\frac{x}{y}\right)^{w}+1}, G(x, y)=1-F(x, y)$ and $H(x, y, z)=x+y+z+A G(x, y)+B F(x, y)$.

This is a strongly non-linear algebraic system of three equations in three unknowns. Even in the simplest case with $w=1$ we would have three coupled fourth degree equations, for which no explicit solutions may be obtained. However, performing a detailed numerical analysis of these equations in order to find their roots (via Newton method for nonlinear equations, for instance) it is observed that the set of steady states $\left(p_{+}^{*}, p_{-}^{*}, p_{0}^{*}\right)$ obtained for each specific 390 choice of parameters (see Fig. 2) is indeed a solution of this system of equations. 